\title{
Actitudes, conocimientos y prácticas de internos de medicina frente a la interrupción voluntaria del embarazo en Medellín, Colombia
}

\author{
Attitudes, knowledge and practices of internal \\ medicine against the voluntary interruption of pregnancy \\ in Medellin Colombia
}

\author{
Juliana Martínez-Sánchez, Laura Trujillo-Numa, Laura Montoya-González, Diana Patricia Restrepo-Bernal \\ 1. Universidad CES, Residente de II año de Psiquiatría \\ 2. Psiquiatra Clínica Las Américas, Medellín \\ 3. Universidad CES, Psiquiatra docente Universidad CES, estudiante de maestría en epidemiología Universidad CES \\ 4. Universidad CES, Médica Psiquiatra de Enlace, docente de pre y post grado Facultad de Medicina, Magíster en Epidemiología, Investigadora coordinadora \\ Grupo Salud Mental, Universidad CES. ORCID iDhttp://orcid.org/0000-0001-8555-6021
}

\begin{abstract}
Resumen
La interrupción voluntaria del embarazo es una opción legal en algunos países bajo circunstancias especiales. Se quiso explorar las actitudes, los conocimientos y las prácticas de internos de medicina frente a la interrupción voluntaria del embarazo en Medellín-Colombia. Se realizó un estudio observacional descriptivo de corte. Se analizaron variables sociodemográficas, actitudes, conocimientos y prácticas frente a la interrupción voluntaria del embarazo. Se realizó un análisis descriptivo de las variables. La información se analizó con el software $\operatorname{SPSS}{ }^{\circledR}$ versión 21.0. Se obtuvo consentimiento informado y aprobación del comité de ética universitario. El principal motivo para realizar la interrupción voluntaria del embarazo, fue por riesgo para la salud mental de la madre. Acerca de la objeción de conciencia el 54,7 \% la tiene, $21 \%$ objetó conciencia durante el pregrado y el 86,7 $\%$ conoce correctamente el proceso a seguir para hacerlo. La totalidad de los encuestados identifica las circunstancias en las cuales el aborto está despenalizado en Colombia, el $96 \%$ conoce el objetivo de la sentencia C355 y el $41 \%$ desconoce que no se establece edad gestacional para practicarla. De los internos que presenciaron IVE el $25,7 \%$ tuvo una repercusión emocional severa con recuerdos frecuentes de lo ocurrido y el $48,7 \%$ tristeza con otras emociones.
\end{abstract}

Palabras claves: aborto terapéutico, actitudes, conocimientos, prácticas, objeción, conciencia

\section{Abstract}

Voluntary Interruption of Pregnancy (VIP) is legal under special circumstances in some countries. The objective of this study was to evaluate the skills, knowledge and attitudes about VIP in medical interns from a private university in Colombia. A cross-sectional study with primary data collection was preformed. A descriptive analysis was done for the variables; socio-demographic, skills, knowledge and attitudes about VIP. The SPSS ${ }^{\circledR}$ software, version 21.0, was used for the statistical analysis. A written consent was signed and approved by the research ethics committee.

The primary circumstance to practice a VIP was if the mother's mental health was at risk. 54,7 \% of the interns endorsed having a conscientious objection and $21 \%$ applied it during their internship year. $86,7 \%$ of the students answered correctly about how to proceed in a VIP case. All of them identified the special circumstances, in which VIP can be done in Colombia, and $96 \%$ recognized the law, but only $41 \%$ knew details about gestational time limits. $25,7 \%$ of the students report having emotional consequences after witnessing a VIP; 48,7 $\%$ presented with depressed mood.

Key Words: abortion, conscientious objection, skills, knowledge and attitudes

\section{Introducción}

En Colombia, la sentencia de la Corte Constitucional C355 emitida en 2006, en el capítulo cuarto, artículo 122, estableció que las mujeres tienen derecho a un aborto legal bajo tres circunstancias específicas: "(i) Cuando la continuación del embarazo constituya peligro para la vida o la salud de la mujer, certificada por un médico; (ii) Cuando exista grave malformación del feto que haga inviable su vida, certificada por un médico; y, (iii) Cuando el embarazo sea el resultado de una conducta, debidamente denunciada, constitutiva de acceso carnal o acto sexual sin consentimiento, abusivo o inseminación artificial o transferencia de óvulo fecundado no consentidas, o de incesto" 1 .

Los estudiantes de medicina conforman un grupo de futuros profesionales que tendrán un papel decisivo frente a la aplicación de las leyes que regulan la interrupción voluntaria del embarazo (IVE) en Colombia. Por tanto la formación académica, así como las posturas personales frente al tema son de gran importancia. Tradicionalmente las actitudes de los médicos frente al aborto están influenciadas por factores diversos como aspectos éticos, religiosos, políticos y dependen además del sexo, la edad o el tipo de práctica de los profesionales de la salud ${ }^{2}$. 
En un estudio reciente con estudiantes de medicina de una universidad colombiana, se identificaron "serias falencias conceptuales" frente a la IVE, con un cambio actitudinal hacia la aceptación de estos procedimientos luego de cursar el semestre de ginecología y obstetricia ${ }^{3}$.

Con base en estos planteamientos se diseñó este estudio con el objetivo de describir las actitudes, los conocimientos y las prácticas de estudiantes de medicina frente a la interrupción voluntaria del embarazo en una universidad privada de Medellín, Colombia.

\section{Metodología}

Estudio observacional transversal con fuente de información primaria. Se incluyeron las siguientes variables: sociodemográficas (edad, sexo), algunas actitudes, conocimientos y prácticas relacionadas con la interrupción voluntaria del embarazo. Para todas las variables de interés se obtuvo la información requerida. La unidad de análisis fueron los internos de medicina de una universidad privada de la ciudad de Medellín, matriculados en el último año de medicina durante el 2015. Se les invitó a participar en la investigación por medio de internet. Aquellos que aceptaron participar y dieron el consentimiento informado, respondieron la encuesta diseñada por los investigadores. Esta incluyó 25 preguntas de selección múltiple y algunas preguntas abiertas de profundización. El lenguaje de las preguntas fue sencillo y para verificar su comprensión se realizó una prueba piloto. La descripción de las variables cualitativas se hizo con frecuencias absolutas y relativas.

Se obtuvo consentimiento informado de todos los participantes. El proyecto fue aprobado por un comité de investigación y por comité de ética institucional.

La información fue analizada con el software SPSS versión 21.0 con licencia amparada.

\section{Resultados}

De 93 internos de medicina matriculados en el 2015, el 56 $\%$ accedió a responder la encuesta. Las edades comprendidas fueron entre 22 y 29 años. El $71,87 \%$ de los encuestados fueron mujeres.

\section{Conocimientos:}

Se exploraron algunos conocimientos frente a la interrupción voluntaria del embarazo.

\section{- Sentencia C355 de 2006}

Cuando se pregunta por el objetivo de la sentencia de la corte al aprobar la IVE, el $96 \%$ responde que se hizo para respetar los derechos de la mujer, el 1,8\% para generar una alternativa legal para las mujeres que tienen embarazos no deseados y el 1,8\% no sabe. Es llamativo que el $41 \%$ de los encuestados dice que la sentencia de la corte estableció en Colombia una edad límite para practicarla, en tanto que el $59 \%$ tiene claro que no se puso un límite de edad gestacional para realizar el procedimiento.

\section{- Aspectos relacionados con el aborto}

Se encontró que el $81 \%$ identifica conceptos claves relacionados con la definición de aborto, el $100 \%$ identifica las circunstancias en las cuales el aborto está despenalizado en Colombia y el $66 \%$ identifica los tratamientos específicos para la IVE según las semanas de gestación. Esto contrasta con el $11 \%$ de internos que identifican la ruta de atención correcta que tiene que seguir la mujer para acceder a la misma.

\section{- Objeción de conciencia}

Se indagaron algunos aspectos que dan cuenta del conocimiento que los internos de medicina podrían tener en relación a la IVE. La totalidad de los encuestados conoce la definición correcta de objeción de conciencia, el 54,7 \% afirmó tener objeción de conciencia frente a la IVE, el 22,6\% no la tiene y el $22,6 \%$ no sabe.

El 86,7\% dice que, si una mujer solicita una IVE a un médico que tiene objeción de conciencia, este debe remitirla de inmediato a otro médico que no tenga la objeción. El 5,6 \% le daría unos días a la mujer para que recapacite y quizás cambie de opinión y el 7,5\% enviaría a la mujer a una evaluación psicológica para ver si tiene problemas mentales.

En cuanto a la objeción de conciencia frente a la IVE, la mayoría (81\%), considera que puede ser interpuesta siempre que un médico así lo determine, el 9,4 \% sólo en algunos casos, el 5,6 \% en ningún caso y el 3, $7 \%$ no sabe.

\section{- Formación recibida en pregrado relacionada con IVE}

Se les pidió a los internos calificar la formación que recibieron en el pregrado frente a la IVE. El 19,0\% considera que fue bueno y el $81,0 \%$ entre regular y muy malo. Un tercio de ellos dice que durante el pregrado se discutió la sentencia C355 que despenalizó el aborto en Colombia, y el resto no está seguro o dice que no se hizo.

\section{Actitudes relacionadas con la IVE:}

Se indagaron algunas actitudes frente a la IVE.

\section{- Repercusión emocional}

35 internos presenciaron una IVE. De ellos el 25,7 \% opinó que la repercusión emocional que había tenido por presenciarla fue severa, el $42,8 \%$ la calificó como moderada, el $22,8 \%$ como leve y el $8,5 \%$ afirmó no tener ninguna repercusión emocional. (Ver figura 1). 

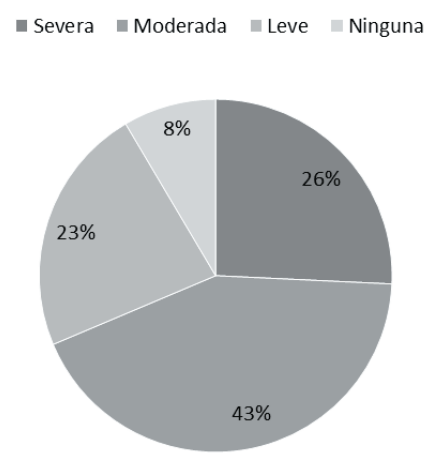

Figura 1. Repercusión emocional de los internos luego de presenciar procedimientos de IVE

Se le solicitó a los internos especificar las emociones que experimentaron luego de presenciar un procedimiento de IVE. Las siguientes emociones fueron incluidas: tristeza, rabia, impotencia, miedo, asco, remordimiento, vergüenza y alegría y otra opción de respuesta fue no haber experimentado ninguna emoción en especial, además se les permitió elegir más de una emoción. (Ver tabla 1).

Tabla 1. Emociones y sentimientos asociados a la los procedimientos de IVE

\section{Emociones y sentimientos}

$\%$

Tristeza con otras emociones

Tristeza

Ninguna emoción

Miedo

Impotencia

5,72

Remordimiento

Alegría

0,0

Ira-rabia

0,0

En cuanto a las consecuencias derivadas de haber presenciado o practicado un procedimiento de IVE, los internos reportaron los siguiente: recuerdos frecuentes de lo ocurrido en un 39,62 $\%$, llanto en un $16,92 \%$ y pesadillas en un $5,64 \%$.

\section{- Presión para presenciar IVE}

Otra de las preguntas hablaba sobre si se habían sentido presionados para presenciar procedimientos de IVE. El 35,5\% dijo que sí y el 64,5\% dijo que no. (Ver figura 2).

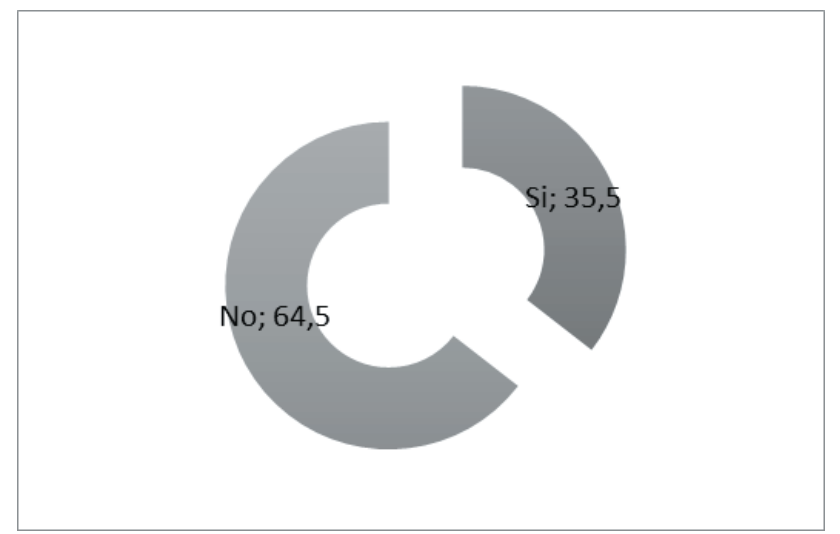

Figura 2. Distribución porcentual de la presión que sintieron los internos para presenciar procedimientos de IVE

\section{Prácticas relacionadas con la IVE:}

Al preguntarles si los profesores de ginecología y obstetricia les habían informado sobre la posibilidad que tenían de objetar conciencia frente a la IVE, el $51 \%$ dijo que sí, el $43 \%$ dice que no y el $6 \%$ no recuerda. Frente a esta misma pregunta, pero con los profesores de pediatría, el $11 \%$ dice que sí, el 66 $\%$ que no y el $23 \%$ no recuerda.

\section{- Objeción de conciencia}

El $21 \%$ de los internos afirmó que había realizado objeción de conciencia, el $74 \%$ no lo hizo y el $5 \%$ dijo no recuerda (Ver figura 3).

De los que objetaron conciencia, el $90 \%$ lo hizo en relación a la atención de una materna y el $10 \%$ por la atención de un neonato. De aquellos que presenciaron IVE el $70 \%$ presenció entre 1 y 3 procedimientos y el $30 \%$ más de tres. De los 15 internos que atendieron neonatos productos de IVE, el $86 \%$ presenció entre 1 y 3 procedimientos y el $14 \%$ más de tres.

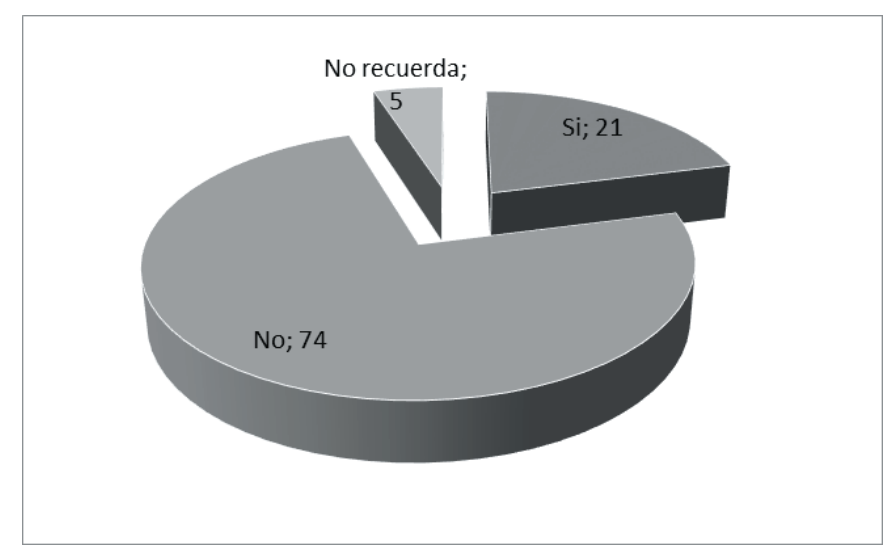

Figura 3. Distribución porcentual de la objeción de conciencia de los internos frente a la IVE 


\section{- Motivos legales para la realización de la IVE}

En cuanto a los motivos legales por los cuales se realizaron las IVE, se encontró que el 52,8 \% de los procedimientos se realizaron por riesgo para la salud mental materna, el 30,5\% por malformaciones fetales, el $5 \%$ por riesgo para la salud física de la madre, y el $11 \%$ por violación (Ver figura 4).

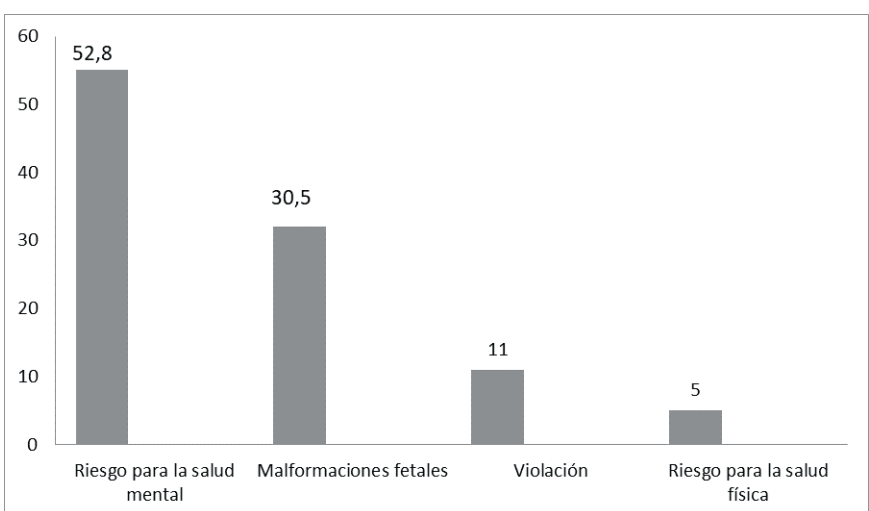

Figura 4. Distribución porcentual de los motivos por los cuales se realizó la IVE

\section{- Actividades realizadas por los internos relacionadas con el procedimiento de IVE}

En cuanto a las actividades que los internos afirman haber realizado en torno a los procedimientos de IVE, se encontraron las siguientes: 1) ordenar en la historia clínica misoprostol o mifespristona vaginal; 2) colocar misoprostol vaginal; 3) atender el parto; 4) cortar el cordón umbilical; 5) intubar al neonato.

\section{Discusión}

Este estudio encontró que uno de cada dos procedimientos de IVE, presenciados por los internos, fueron realizados por riesgo para la salud mental de la madre. Esta cifra supera las estadísticas nacionales donde sólo el $12 \%$ correspondía a esta causal ${ }^{4}$.

Otro hallazgo de importancia se relaciona con la objeción de conciencia. Uno de cada cinco estudiantes encuestados objetó conciencia. Esto coincide con un estudio noruego, el cual encontró que el $19 \%$ de los estudiantes de medicina encuestados tenían objeción de conciencia para procedimientos de aborto ${ }^{5}$.

Frente a la objeción de conciencia, existe un conflicto entre el deber ante la ley y el deber ante la propia conciencia. Por esto motivo se permite el derecho a resistir los mandatos de la autoridad cuando se va en contra de los principios morales del individuo. La objeción de conciencia es un derecho basado en las creencias más profundas del ser y cuyo fundamento ético es la libertad ideológica ${ }^{6}$.

Los problemas frente a este derecho de los estudiantes surge, no cuando el estudiante efectúa el razonamiento práctico, sino cuando pretende comportarse de acuerdo con la opción escogida. Una investigación con estudiantes de medicina peruanos encontró que a pesar de contar con una Ley Universitaria que asegura la libertad del estudiante y el pluralismo, aquellos que interponían objeción de conciencia: "han sido discriminados o tuvieron que sufrir consecuencias a veces graves, en su desempeño académico y ejercicio profesional". Según algunos autores, el problema es que una persona joven se está enfrenatndo con situaciones para las cuales no está preparado o no tiene la formación suficiente ${ }^{7}$.

En la literatura se reportan dos razones principales en los profesionales de la salud para objetar conciencia. En el primer caso se debe a desconocimientos teóricos y prácticos en el ejercicio médico y en el segundo caso se presenta por motivos personales como creencias, principios o convicciones. Con respecto a las razones, algunos autores afirman que la primera obligación debe ser con los pacientes y no con los propios miembros del personal. Por tanto, se ha sugerido que los objetores deben estar obligados a justificar su posición, "probando la autenticidad", ya que el libre acceso a la objeción de conciencia podría llevar al personal a negarse para evitar deberes desagradables ${ }^{8}$.

López et al., exploraron las actitudes de estudiantes españoles de podiatría y encontraron que la mitad de ellos creía que tenían el derecho de objetar conciencia frente a cualquier procedimiento clínico. Esta objeción se asoció con preferencias religiosas ${ }^{9}$.

En cuanto a los conocimientos teóricos relacionados con el aborto, casi la totalidad de los encuestados identificaron conceptos claves del mismo. Cabe resaltar que frente a la sentencia c355 de despenalización del aborto emitida en el año 2006, la totalidad de los encuestados identificaron las circunstancias en las cuales el aborto está despenalizado en Colombia. Por el contrario, solo la minoría identificó la ruta de atención adecuada que debe seguir la mujer para tener acceso al procedimiento de IVE. Además, un gran porcentaje de los encuestados respondieron que la Sentencia establece una edad gestacional límite para practicar la IVE, lo cual no es cierto y genera confusión. En un estudio que envió una encuesta por correo a los 126 programas de ginecología y obstetricia de Estados Unidos, en la cual se indagaba por la educación relacionada con el aborto que se le suministraba a los estudiantes de pregrado de medicina, se concluyó que al 45 $\%$ de los estudiantes se les ofrecía una experiencia clínica. La participación generalmente era baja y la mitad de las escuelas médicas ofrecían en el cuarto año un curso electivo de salud reproductiva, pero pocos estudiantes participaban ${ }^{10}$.

Con respecto a las emociones relatadas por los encuestados, se observa un predominio de la tristeza y otras emociones negativas sin ningún reporte de alegría o sentimientos positivos . Estos hallazgos contrastan con los de Espey et al, los cuales realizaron un estudio en la Universidad de Nuevo México en el cual participaron 86 estudiantes (68\%) de 145 que rotaron por ginecología y obstetricia en el período académico. 
La mayoría de ellos refirió que participar en las sesiones de asesoría a mujeres que deseaban el aborto o presenciar estos procedimientos, había sido "una de las mejores experiencias". El $38 \%$ reportó que, tras la experiencia clínica, experimentaron cambios en las actitudes respecto al aborto (de mayor apoyo) y el $94 \%$ refirió sentirse "más solidario" frente al acceso de las mujeres a los servicios de aborto ${ }^{11}$.

Este estudio debe ser analizado a la luz de las siguientes limitaciones. Primero, los resultados están basados en una encuesta, la cual es susceptible de ser influida por sesgos de memoria. Segundo, es posible que los participantes no brindaran información verás respecto a temas sensibles como los tratados en el estudio. Tercero, sólo se tiene información de los internos que aceptaron participar de la encuesta, por lo tanto los resultados no pueden ser generalizados. Y cuarto las opiniones de los estudiantes pueden cambiar en el tiempo y aquellos que tienen objeción de conciencia frente a la IVE pueden en el futuro no ejercer este derecho y viceversa. No obstante estas limitaciones, este estudio, presenta informaciòn valiosa, frente a las actitudes, conocimientos y prácticas de algunos internos de medicina frente a la IVE en el contexto colombiano.

\section{Conclusiones}

El principal hallazgo de este investigación fue que uno de cada dos estudiantes de medicina encuestados manifestó tener objeción de conciencia frente a la IVE, pero solo uno de cada cinco la interpuso durante el pregrado. Además, tres de cada cuatro estudiantes calificó la repercusión emocional luego de presenciar estos procedimientos entre severa y moderada, con predominio de tristeza combinada con otras emociones y sentimientos negativos.

Se comprobó también que hay desconocimiento frente a algunos aspectos de la IVE. Esto refleja la necesidad de reforzar los conocimientos sobre la legislación colombiana y profundizar en la formación teórica y práctica de esta intervención y todo lo que trae consigo. De esta manera se podría lograr que los estudiantes estén capacitados, logren tomar decisiones y realizar acciones autónomas, que les ayuden a prevenir repercusiones de tipo moral y emocional.

Como es de esperar, frente a una normativa reciente como es la sentencia c355, los mecanismos académicos y legales que regulan la objeción de conciencia en estudiantes de medicina no están establecidos, frente a lo cual las universidades están llamadas a generar reflexión y propiciar mecanismos que resuelvan este importante vacío.

\section{Referencias Bibliográficas}

1. Cardozo de Martínez, C. A. "Implicaciones éticas, jurídicas y médicas de la sentencia C-355 de la Corte Constitucional: un avance para el ejercicio de los derechos humanos, sexuales y reproductivos de las colombianas". Ministerio de la Protección Social de Colombia. Bogotá: Editorial Kimpres; 2007. ISBN
978-958.

2. González de León Aguirre D, Salinas Urbina AA. Los médicos en formación y el aborto: opinión de estudiantes de medicina en la ciudad de México. Cad. Saúde Pública. 1997;13(2):227-36.

3. Quintero-Roa EM, Ochoa ME. Conocimientos y actitudes de estudiantes de medicina ante el aborto inducido despenalizado. Rev. Salud pública. 2015;17(6):912-24.

4. Alcaldía de Medellín [internet]. Gloria Stella Penagos Velásquez. El aborto en colombia un problema social, de salud pública y de salud de las mujeres. Disponible en: http://www.medellin.gov.co

5. Nordstrand SJ, Nordstrand MA, Nortvedt P, Magelssen M. Medical students' attitudes towards conscientious objection: a survey. J. Med Ethics. 2014;40(9):612-4.

6. Salas SP, Besio M, Bórquez Estefó G, Salinas RA, Valenzuela CY, Micolich C, et al. Position paper from the Department of Ethics of the Chilean College of Physicians about conscientious objection. Rev Med Chil. 2016;144(3):382-7.

7. Morales-Guzmán-Barrón R. La objeción de conciencia y el estudiante de medicina. Rev. Soc Peru Med Interna. 2008;21(2):78-81.

8. Kantymir L, McLeod C. Justification for Conscience Exemptions in Health Care: Justification for Conscience Exemptions in Health Care. Bioethics. 2014;28(1):16-23.

9. López D, de la Campa-Portela RM, Losa-iglesias ME, Ramos-Gal J, Munuera-Martínez P, GarcíaSánchez M, et al. Concientious objection in spanish podiatrist students: a questionnaire survey. Acta Bioethica. 2016;22 (2):341-5.

10. Espey E, Ogburn T, Chavez A, Qualls C, Leyba M. Abortion education in medical schools: a national survey. Am J of Obstet and Gynecol. 2015;192:6403.

11. Espey E, Ogburn T, Dorman, F. Student attitudes about a clinical experience in abortion care during the obstetrics and gynecology clerkship. Academic Medicine. 2004;79(1):96-100. 